\title{
DOCUMENTS
}

Joachim Eichler

\section{PARTEI UND GEWERKSCHAFT: EIN BRIEFWECHSEL IM VORFELD DES SPD-PARTEITAGS JENA 1905}

Im Jahr 1905 wurde ein tiefgreifender Dissens zwischen der Führung der Sozialdemokratischen Partei Deutschlands und den Leitern der sozialdemokratisch orientierten Freien Gewerkschaften offenbar. Die in diesem Jahr beginnenden öffentlichen Auseinandersetzungen galten vor allem der Form der Maifeier sowie der Diskussion des politischen Massenstreiks. Zum Verständnis der nachstehend veröffentlichten Briefe ist es erforderlich, die Entstehung dieses politischen Streits zu berücksichtigen. Diese soll hier zunächst skizziert werden.

Die seit dem Pariser Kongre $\beta$ der Internationale 1889 propagierte Arbeitsruhe am 1. Mai als Demonstration für den achtstündigen Arbeitstag, Arbeiterschutz und Arbeiterrechte wurde in Deutschland zunehmend praktiziert, ${ }^{1}$ was wegen der damit verbundenen Gegenmaßnahmen der Unternehmer bald kritische Überlegungen in den Gewerkschaftsvorständen hervorrief, ob der Effekt der Maifeier durch Arbeitsruhe den damit verbundenen organisatorischen und finanziellen Aufwand lohne. Nachdem eine vertrauliche Besprechung über diese Frage auf dem Stuttgarter Gewerkschaftskongreß 1902 noch ohne konkretes Ergebnis geblieben war, ${ }^{2}$ wurde dann in der Konferenz der Gewerkschaftsvorstände im Oktober 1903 beschlossen, beim bevorstehenden Kongre $\beta$ der Internationale in Amsterdam eine Revision des Pariser Maifeierbeschlusses anzustreben. Die Delegierten der deutschen Gewerkschaften sollten sich um einen Beschluß bemühen, , , daß am 1. Mai die Demonstration in Versammlungen stattfindet, die am Abend veranstaltet werden, oder sonst eine Form der Demonstration bestimmt wird, die von jeder Durchführung der Arbeits-

1 Vgl. Protokoll über die Verhandlungen des Parteitags der Sozialdemokratischen Partei Deutschlands, abgehalten zu Jena vom 17. bis 23. September 1905, S. 238.

2 Vgl. Protokoll der Konferenz der Vorstände der Centralverbände, abgehalten im Berliner Gewerkschaftshaus am Montag den 12. und Dienstag den 13. Oktober 1903, S. 15 . 
ruhe absieht". ${ }^{3}$ Die Vertreter der Gewerkschaftsvorstände bezweifelten mehrheitlich, daß die Unterstützungszahlungen an die wegen der Maifeier ausgesperrten Arbeiter sinnvoll angelegtes Geld wären. ${ }^{4}$ Die Generalkommission der Gewerkschaften Deutschlands wollte in Verhandlungen erreichen, daß sich der Parteivorstand der SPD dieser Auffassung anschloß. In zwei gemeinsamen Sitzungen von Parteivorstand und Generalkommission stellte sich heraus, daß in dieser Frage keine Einigung erreicht werden konnte. ${ }^{5}$ Während die Generalkommission eine Maifeier-Resolution im oben genannten Sinne entworfen hatte, ${ }^{6}$ legte die Parteiführung dem Amsterdamer Kongreß eine Resolution vor, die auf Ausbau der Arbeitsruhe

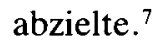

Die Entscheidung darüber, welche dieser Resolutionen in Amsterdam als Stimme der deutschen Arbeiterbewegung präsentiert werden sollte, überließen die beiden Führungsgremien der deutschen Delegation zum Kongreß, die sich dann mit 36 gegen 20 Stimmen für die Resolution des Parteivorstands entschied. ${ }^{8}$ Diese Resolution wurde vom Kongre $\beta$ leicht geändert angenommen ${ }^{9}$ und anschließend vom Bremer Parteitag der SPD bekräftigt. ${ }^{10} \mathrm{Bis}$ zu diesem Zeitpunkt war die Maifeierdebatte in der deutschen Arbeiterbewegung eine Angelegenheit der obersten Funktionärsebene geblieben, über die Vertraulichkeit gewahrt werden sollte. ${ }^{11}$ Auf der nächsten Konferenz von Generalkommission und Gewerkschaftsvorständen fand dann die Auffassung eine Mehrheit, daß es für die deutschen Gewerkschaften angesichts der jüngsten Beschlüsse nötig wäre, Farbe zu bekennen und den gewerkschaftlichen Standpunkt zur Maifeier auf dem Kölner Gewerkschaftskongreß deutlich zu machen. ${ }^{12}$ Während der Vorsitzende der Generalkommission, Carl Legien, sich gegen die Behandlung

3 Ebd., S. 18.

4 Ebd., S. 16.

5 Correspondenzblatt der Generalkommission der Gewerkschaften Deutschlands, Jg. 14 (1904), S. 595.

6 Zitiert in Th. Leipart, ,Die Gewerkschaften und die Maifeier”, in: Sozialistische Monatshefte, Jg. 9 (1905), Bd 1, S. 407-412, S. 412.

7 Correspondenzblatt, Jg. 14, S. 596.

8 Ebd., S. 595.

9 Ebd., S. 596.

10 Protokoll über die Verhandlungen des Parteitags der Sozialdemokratischen Partei Deutschlands, abgehalten zu Bremen vom 18, bis 24. September 1904, S. 287.

11 Nachdem Max Quarck in der Frankfurter Volksstimme über die internen Verhandlungen der deutschen Delegation in Amsterdam berichtet hatte, warf ihm das Correspondenzblatt vor, gegen die vereinbarte Vertraulichkeit verstoßen zu haben. Correspondenzblatt, Jg. 14, S. 595.

12 Protokoll der Konferenz der Vorstände der Centralverbände, abgehalten im Berliner Gewerkschaftshaus am Montag 24., Dienstag 25. und Mittwoch 26. Oktober 1904, S. 7. 
der Maifeierfrage auf dem Gewerkschaftskongreß aussprach, weil daraus ein Scheinkonflikt zwischen der Partei und der Gewerkschaft entstehen könne, ${ }^{13}$ war die Mehrkeit der Ansicht, daß man - ohne dem Parteitagsbeschluß zu widersprechen - für eine Änderung der Form der Maifeier arbeiten könne. Als Ansatzpunkt dafür sah man die Einschränkung der Amsterdamer Resolution, daß die Arbeitsruhe überall dort durchgeführt werden solle, ,wo es ohne Schädigung der Arbeiterinteressen möglich ist". ${ }^{14}$ Die Gewerkschaftsführer wollten den Begriff der ,,Schädigung” auf dem Kölner Kongreß genauer definieren ,, und auch daraufhin arbeiten, $\mathrm{da} ß$ der nächste internationale Kongreß einen anderen Beschluß faßt als in diesem Jahre". ${ }^{15}$

In der gleichen Konferenz wurde einstimmig beschlossen, das Thema ,Generalstreik oder politischer Massenstreik” auf die Tagesordnung des Kölner Kongresses zu setzen. Ähnlich wie bei der Maifeierfrage war dies eine Reaktion auf eine Resolution des Internationalen Arbeiterkongresses in Amsterdam. ${ }^{16}$ Die Frage des Generalstreiks als Kampfmittel der deutschen Arbeiterbewegung war zuvor immer wieder theoretisch erörtert worden, ohne dabei eine nennenswerte Resonanz zu erreichen. Bestimmend dabei war, daß Friedrich Engels den anarchistischen Generalstreik abgelehnt hatte. Rosa Luxemburg faßte die Engels'sche Position wie folgt zusammen: ,,Entweder ist das gesamte Proletariat noch nicht im Besitz mächtiger Organisationen und Kassen, dann kann es den Generalstreik nicht durchführen, oder es ist bereits mächtig genug organisiert, dann braucht es den Generalstreik nicht." 17 Den Internationalen Arbeiterkongressen jedoch wurde die Frage des Generalstreiks immer wieder vorgelegt. Auf den Kongressen 1889, 1891, 1893, 1896 und 1900 wurden Resolutionen, die den absoluten Generalstreik befürworteten, abgelehnt bzw. nicht behandelt. ${ }^{18}$ Neben der ständigen Generalstreik-Agitation der Anarchisten waren es vor allem die tatsächlich stattfindenden politischen Streiks in Europa, die die Frage des Generalstreiks immer wieder auf der Tagesordnung der Internationale erscheinen ließen. Die Massenstreiks in Belgien 1891, 1893 und 1903, in Österreich 1902, in Holland 1903 und Italien 1904 erhielten diese Frage aktuell. ${ }^{19}$ Beim Internationalen Arbeiterkon-

13 Ebd., S. 5.

14 Ebd., S. 4.

15 Ebd.

16 Diese ist zitiert in Correspondenzblatt, Jg. 14, S. 573.

17 R. Luxemburg, Massenstreik, Partei und Gewerkschaften, in Politische Schriften, Bd 1, Frankfurt/M. 1966, S. 136.

18 Protokoll Jena, S. 302 f.; vgl. auch E. Lüdke, Müssen wir die Idee des Generalstreiks propagieren? Mit einem Vorwort von H. Roland-Holst, Berlin 1910, S. $7 \mathrm{f}$.

19 Vgl. Die Massenstreikdebatte, hrsg. von A. Grunenberg, Frankfurt/M. 1970, S. 10ff.; vgl. auch Protokoll Jena, S. 305f. 
greß in Amsterdam 1904 wurde erneut über den Generalstreik beraten. Die schließlich angenommene Resolution lehnte diesen zwar ab, erklärte aber den politischen Massenstreik, ,, der sich über einzelne, für das Wirtschaftsleben wichtige Betriebszweige ausdehnt", ${ }^{20} \mathrm{zu}$ einem unter bestimmten Umständen tauglichen Kampfmittel des Proletariats. Unter der Voraussetzung starker Arbeiterorganisationen und guter Vorbereitung der Aktion ${ }^{21}$ sei der politische Massenstreik ,,ein äußerstes Mittel [. . .] um bedeutende gesellschaftliche Veränderungen durchzuführen oder sich reaktionären Anschlägen auf die Rechte der Arbeiter zu widersetzen". ${ }^{22}$ In der bereits erwähnten Konferenz der Gewerkschaftsvorstände im Oktober 1904 wurde daraufhin beschlossen, die Frage ,,Generalstreik oder politischer Massenstreik" auf die Tagesordnung des Kölner Gewerkschaftskongresses zu setzen, ${ }^{23}$ da auch der Bremer SPD-Parteitag deutlich gemacht hatte, da $B$ dieses Thema auf dem folgenden Parteitag behandelt werden sollte. ${ }^{24}$

Die Gewerkschaftsvorstände waren der Ansicht, daß in Streikfragen erfahrene Praktiker ungleich berufener als die Theoretiker seien, über die Frage des Generalstreiks zu reden. Deshalb wollten sie das Thema noch vor dem Parteitag behandeln. ${ }^{25}$ Es wurde ausdrücklich beschlossen, daß als Referent dazu nur ein Gegner des Generalstreiks ausgewählt werden sollte. ${ }^{26}$ Während die unterschiedlichen Auffassungen der Maifeier beiden Führungsgremien der Arbeiterbewegung seit der Vorbereitung des Amsterdamer Kongresses bekannt waren, war der Dissens in der Massenstreikfrage bis zum Kölner Gewerkschaftskongreß noch nicht deutlich. Zwar war auf dem Bremer Parteitag beantragt worden, den Generalstreik bzw. politischen Massenstreik auf die Tagesordnung des Jenaer Parteitages zu setzen, es war dies aber nicht beschlossen, sondern dem Parteivorstand ,,zur Erwägung" überwiesen worden. ${ }^{27}$ Eine ausführliche Diskussion des Massenstreiks hatte in der Partei noch nicht stattgefunden, die theoretischen Erörterungen des Themas ${ }^{28}$ zeigten sich sehr uneinheitlich. So orientierten sich manche Autoren an der anarchistischen Vorstellung vom

20 Zitiert nach Correspondenzblatt, Jg. 14, S. 573.

21 Vgl. ebd., S. 572.

22 Ebd., S. 573.

23 Protokoll Vorständekonferenz 1904, S. 7.

24 Protokoll Bremen, S. 190-98.

25 Protokoll Vorständekonferenz 1904, S. 7.

27 Protokoll Bremen, S. 198.

28 Vgl. W. Düwell, ,,Zur Frage des Generalstreiks", in: Neue Zeit, Jg. 23 (1904-05), Bd 1, S. 248-54; E. Umrath, ,Zur Generalstreikdebatte”, ebd., Bd 2, S. 13-20; E. Kloth, ,,Generalstreik und Maifeier auf dem Gewerkschaftskongreß in Köln", ebd., S. 215-20; E. Bernstein, ,Ist der politische Streik in Deutschland möglich", in: Sozialistische Monatshefte, Jg. 9, Bd 1, S. 29-37. 
Generalstreik, andere an der Amsterdamer Generalstreik-Resolution. Die Terminologie war uneinheitlich, die Autoren verwandten die Begriffe ,,Generalstreik”, , ,Massenstreik”, ,,politischer Massenstreik” und ,,politischer Streik". Eindeutig war zu Beginn des Gewerkschaftskongresses nur, $\mathrm{da} B$ es Kräfte in der Partei und auch in den Gewerkschaften ${ }^{29}$ gab, die den politischen Massenstreik als zumindest erwägenswert ansahen. Henriette Roland-Holsts Schrift über den politischen Massenstreik, ${ }^{30}$ die die Massenstreikdebatte vorantrieb und vereinheitlichte, die auch Kautskys ${ }^{31}$ und Bebels ${ }^{32}$ Einstellung zum Massenstreik beeinflußte, erschien erst im Monat nach dem Gewerkschaftskongre $B .{ }^{33}$

Auf dem Kölner Kongre $\beta$ trat als Referent zum Generalstreik Theodor Bömelburg auf. ${ }^{34}$ Die von ihm vorgelegte Resolution lehnte die Propagierung des politischen Massenstreiks ab, weil sich die Arbeiterschaft nicht eine bestimmte Taktik vorschreiben lassen könne. Allen Versuchen, den Massenstreik zu propagieren, sollten die Arbeiter energisch entgegentreten. ${ }^{35}$ Bömelburg warf den Theoretikern vor, durch die Behandlung der Generalstreikfrage Unruhe in die Arbeiterbewegung gebracht zu haben; ,Um aber unsere Organisationen auszubauen, dazu bedürfen wir in der Arbeiterbewegung Ruhe." 36 Auf den Vorhalt, man hätte sich über den Generalstreik mit der Partei verständigen sollen, stellte er die Motivation für die Behandlung des Themas klar: ,Weil doch schließlich beim Streik die Gewerkschaft die Hauptlast trägt, haben wir geglaubt, daß der Gewerkschaftskongre $ß$ zuerst entscheiden müsse. ${ }^{\prime 37}$ Bei der Abstimmung über die Resolution wurde der Absatz, der neben dem anarchistischen Generalstreik auch die Propagierung des politischen Massenstreiks verwarf, von einer Minorität von 30 Delegierten ${ }^{38}$ abgelehnt, die Resolution im ganzen

29 Vgl. Kloth, ,Generalstreik und Maifeier”. Kloth war Vorstandsmitglied der Buchbindergewerkschaft.

30 H. Roland-Holst, Generalstreik und Sozialdemokratie. Mit einem Vorwort von Karl Kautsky, Dresden 1905.

31 K. Kautsky, ,,Die Folgen des japanischen Sieges und die Sozialdemokratie”, 3. Teil, in: Neue Zeit, Jg. 23, Bd 2, S. 494.

32 Bebel an Adler, 16. September 1905, in Victor Adlers Briefwechsel mit August Bebel und Karl Kautsky, hrsg. von F. Adler, Wien 1954, S. 468.

33. Vgl. Vorwärts, Jg. 22 (1905), Nr 146, S. 5.

34 Bömelburg war Vorstandsmitglied der Maurergewerkschaft und SPD-Reichstagsabgeordneter.

35 Protokoll der Verhandlungen des fünften Kongresses der Gewerkschaften Deutschlands, abgehalten zu Köln a.Rh. vom 22. bis 27. Mai 1905, S. 30.

36 Ebd., S. 221.

37 Zitiert nach Vorwärts, Jg. 22, Nr 124, S. 9. Im offiziellen Protokoll der Generalkommission fehlt dieser Satz, Protokoll Köln, S. 228.

38 Vorwärts, a.a.O. Die genaue Zahl der anwesenden Delegierten an diesem Verhandlungstag ließ sich nicht ermitteln, am Tag zuvor waren 199 stimmberechtigte Delegierte 
wurde dann gegen 7 Stimmen angenommen. ${ }^{39}$ Zum Punkt ,Maifeier" sprach auf dem Kongreß Robert Schmidt, dessen vorgelegte Resolution für eine Verlegung der Maifeier auf den Abend des 1. Mai eintrat. ${ }^{40}$ Seine Begründung folgte der bereits geschilderten Argumentationslinie. ${ }^{41}$ Die Diskussion um diese Resolution gestaltete sich sehr heftig. Während einzelne Delegierte die Maifeier an sich ablehnten, sprachen andere dafür, weiterhin die Arbeitsruhe durchzuführen..$^{42}$ Der Resolution Schmidt wurde eine Resolution im Sinne des Amsterdamer Maifeierbeschlusses entgegengestellt, ein dritter Antrag wollte die Generalkommission beauftragen, über die Frage der Maifeier mit dem Parteivorstand zu verhandeln. ${ }^{43}$ Nachdem die scharf geführte Debatte, die eine knapp verlaufende Kampfabstimmung möglich erscheinen ließ, am Abend des fünften Verhandlungstages abgebrochen worden war, überraschte Schmidt die Delegierten am folgenden Vormittag mit der Erklärung, alle Resolutionen würden zurückgezogen. ${ }^{44}$ Er begründete dies damit, daß man aus einer Fortführung der Debatte und einer folgenden Kampfabstimmung keinen Konflikt zwischen Partei und Gewerkschaft entstehen lassen wolle. ${ }^{45}$ Der Vorsitzende Bömelburg schloß die Debatte mit der Bemerkung, da $ß$ nun der Maifeier-Beschluß des Amsterdamer Kongresses maßgebend bleibe, daß der Kölner Kongreß jedoch Generalkommission und Parteivorstand auffordere, sich noch vor dem nächsten internationalen Kongreß auf eine Linie in der Maifeierfrage zu einigen. ${ }^{46}$

Nach Abschluß des Kongresses resümierte der Vorwärts, daß sich auf diesem ein Riß in der Arbeiterbewegung gezeigt habe, der aber nicht zwischen Partei und Gewerkschaft, sondern - wie die heftigen Debatten zeigten - quer durch die Gewerkschaften verlaufe. ${ }^{47}$ Vor allem den oft unbeherrschten Ton der Streitgespräche kritisierte der Vorwärts, zeigte sich aber in Bezug auf die Folgen für das Verhältnis Partei-Gewerkschaft sehr gelassen. ${ }^{48}$ Eine vordergründige Gelassenheit zeigten auch die meisten

anwesend, Protokoll Köln, S. 215.

39 Protokoll Köln, S. 229.

40 Ebd., S. 31f.

41 Ebd., S. 229ff.

42 Ebd., S. 233-41.

43 Ebd., S. 32.

44 Ebd. S. 245f. Laut Vorwärts-Kommentar, ,ging nach dem ersten Augenblick fassungsloser Überraschung [. . . ] ein Aufatmen durch die Masse der Delegierten." Vorwärts, Jg. 22, Nr 124, S. 1.

45 Protokoll Köln, S. 246.

46 Ebd., S. 248.

47 Vorwärts, Jg. 22, Nr 124, S. 1f.

48 Ebd., sowie Nr 132, S. 1. 
Kommentare der Parteipresse, die im Vorwärts zitiert wurden, mit der Behandlung der Massenstreik- und der Maifeierfrage waren die gleichen Kommentare jedoch unzufrieden. Der Maifeier sei ein ,schwere[r] Schlag" versetzt worden, ${ }^{49}$ auch habe niemand die Gewerkschaften auf die Taktik des Massenstreiks festlegen wollen, insofern hätte man die Behandlung dieser Frage besser unterlassen..$^{50}$ Karl Kautskys Kritik am Kölner Kongreß bezog sich vor allem auf die Absage an den politischen Massenstreik. In der Neuen Zeit kritisierte er das Referat Bömelburgs, das nach seiner Ansicht in keiner Weise der Amsterdamer Massenstreikresolution gerecht geworden war. ${ }^{51}$ Als Ursache für die Abiehnung des Massenstreiks sah Kautsky das von Bömelburg angesprochene ,,Ruhebedürfnis" der Gewerkschaften, ${ }^{52}$ gegen das er scharf polemisierte. ${ }^{53}$

Auch innerhalb der Gewerkschaften wurden kritische Stimmen laut. Die Gewerkschaft, das Organ der österreichischen Generalkommission, nannte das Vorgehen der deutschen Gewerkschaftsführung in den Fragen Maifeier und Massenstreik einen ,,schwere[n] Fehler". ${ }^{54}$ Die im Juni 1905 zusammentretende Generalversammlung des Metallarbeiter-Verbands, der mit 212000 Mitgliedern größten deutschen Gewerkschaft, ${ }^{55}$ beschloß eine für die Arbeitsruhe eintretende Maifeier-Resolution, die ein Tadelsvotum an die Kongreßdelegierten des Verbandes enthielt, weil diese in Köln gegen die Arbeitsruhe am 1. Mai gesprochen und für die Massenstreikresolution Bömelburgs votiert hatten. ${ }^{56}$ Im gleichen Monat trat die Generalversammlung des Bergarbeiter-Verbandes ${ }^{57}$ zusammen. Hier wurde der Kongreßdelegierte Leimpeters wegen seiner auf dem Kongreß gezeigten extremen Ablehnung der Arbeitsruhe ${ }^{58}$ scharf kritisiert, ${ }^{59}$ einer beantragten Verur-

49 So die Schwäbische Tagwacht, zitiert nach Vorwärts, Jg. 22, Nr 129, S. 9.

50 Vorwärts, Jg. 22, Nr 126, S. 4; Nr 129, S. 9; Nr 132, S. 1.

51 Vgl. K. Kautsky, ,,Der Kongreß von Köln”, in: Neue Zeit, Jg. 23, Bd 2, S. 311.

52 Siehe oben, S. 73. Es sei hier darauf hingewiesen, daß Kautsky sich bei seiner Polemik offensichtlich auf das Kongreßprotokoll des Vorwärts stützte, in dem das entsprechende Wort Bömelburgs durch Verkürzung einen anderen Inhalt erhielt als in der hier zitierten Fassung des offiziellen Protokolls. Vgl. Kautsky, ,,Der Kongreß von Köln”, S. 313; vgl. Vorwärts, Jg. 22, Nr 123, S. 9.

53 Kautsky, ,Der Kongreß von Köln”, S. 313ff.

54 Vorwärts, Jg., 22, Nr 137, S. 4.

55 Stand Ende Juni 1905, vgl. Correspondenzblatt, Jg. 15 (1905), S. 526.

56 Metallarbeiter-Zeitung. Organ für die Interessen der Metallarbeiter, Jg. 23 (1905), Nr 27, S. 210.

57 Der ,,Alte Verband" hatte Ende Juni 130000 Mitglieder und war damit die zweitgrößte deutsche Gewerkschaft, siehe Anm. 55.

$58 \mathrm{Vgl}$. Protokoll Köln, S. 239.

59 Vorwärts, Jg. 22, Nr 136, S. 6. 
teilung Leimpeters' stimmte jedoch nur ein Drittel der Delegierten zu. ${ }^{60}$ Auch auf der Generalversammlung des Holzarbeiterverbandes ${ }^{61}$ wurde die Kölner Maifeierdebatte kritisiert. Robert Schmidt - als Holzarbeiter Verbandsmitglied - wurde wegen der von ihm vertretenen Maifeierresolution angegriffen.$^{62} \mathrm{Im}$ Juliheft der Sozialistischen Monatshefte übte dann der Gewerkschaftler v. Elm ${ }^{63}$ an den Verhandlungen des Kölner Kongresses Kritik. Die Massenstreikresolution nannte er ein ,,Maulkorbgesetz in schlimmster Form", ${ }^{64}$ die Maifeier durch Arbeitsruhe verteidigte er als eingebürgertes Demonstrationsmittel, das wirksamer sei als ,, noch so viele Tausende von Resolutionen, die man in Versammlungen beschließt" ${ }_{65}$

Anfang Juli 1905 wurde dann die vom Parteivorstand aufgestellte Tagesordnung des Jenaer Parteitags bekannt, ${ }^{66}$ auf der auch die beiden umstrittenen Themen standen. Als Referent zum Thema ,Maifeier" wurde Richard Fischer benannt, der schon auf dem Bremer Parteitag über dieses Thema referiert und dabei die Amsterdamer Resolution befürwortet hatte. ${ }^{67} \mathrm{Zum}$ Tagesordnungspunkt ,,Der politische Massenstreik und die Sozialdemokratie" sollte August Bebel sprechen. Während in der Maifeierfrage allein aus der Auswahl des Referenten der Plan gefolgert werden konnte, den Absichten der Generalkommission zu widersprechen, waren die Pläne des Parteivorstands in Sachen Massenstreik noch nicht abzusehen. Angesichts der heftigen Reaktionen auf den Kölner Kongreß mußte die Generalkommission jedoch befürchten, daß es auf dem Parteitag zu einem ernsten Konflikt zwischen Partei und Gewerkschaft kommen werde. Es gab Erwartungen, daß die deutsche Arbeiterbewegung sich auf dem Jenaer Parteitag selbst ein , Jena" ${ }^{68}$ bereiten werde.

60 Ebd., Nr 137, S. 5.

61 Die Gewerkschaft der Holzarbeiter war mit über 114000 Mitgliedern die drittgrößte im Deutschen Reich, siehe Anm. 55.

62 Vorwärts, Jg. 22, $\mathrm{Nr} 145, \mathrm{~S} .6$.

${ }_{63}$ Adolph von Elm, Tabakarbeiter aus Hamburg, gehörte schon auf dem Gewerkschaftskongreß zu den wenigen Opponenten gegen die Massenstreikresolution der Generalkommission. Seine Stimme wurde deshalb besonders gehört, weil er 1890 Gründungsmitglied der Generalkommission gewesen war, der er bis 1896 angehörte. Vgl. Protokoll Köln, S. 226f.; K. Schönhoven, Expansion und Konzentration, Stuttgart 1980, S. $264 \mathrm{ff} ., 300$.

${ }_{64}$ A. v. Elm, ,,Rückblick auf den Fünften Deutschen Gewerkschafts-Kongreß”, in: Sozialistische Monatshefte, Jg. 9. Bd 2, S. 569.

is Ebd., S. 575.

to Vgl. Vorwärts, Jg. 22, Nr 155, S. 1.

67 Protokoll Bremen, S. 275ff.

68 Eine Anspielung auf die Schlacht bei Jena vom 14. Oktober 1806. Vgl. Protokoll Jena, S. 151: ,,Wer auf ein Jena hofft, der hofft als ein Narr." 
Diese Möglichkeit klein zu halten, indem Partei- und Gewerkschaftsführung gemeinsam Kompromißformeln suchen, war das Anliegen, das dem hier veröffentlichten Brief von Robert Schmidt an August Bebel zugrunde lag. ${ }^{69}$ Robert Schmidt (1864-1943) war gelernter Klavierbauer und durch die Holzarbeitergewerkschaft zur deutschen Arbeiterbewegung gestoßen. Nachdem er als Arbeitersekretär gearbeitet hatte, wurde er 1893 Redakteur des Vorwärts, was er bis 1903 blieb. In diesem Jahr wurde er für die SPD in den Reichstag gewählt, dessen Mitglied er bis zum Ende des Kaiserreichs blieb. Er vertrat dort den fünften Berliner Wahlkreis. Nachdem Schmidt in den frühen neunziger Jahren Sympathien für die Bewegung der ,Jungen" gezeigt hatte,$^{70}$ verstärkte er sein gewerkschaftliches Engagement und wurde 1902 in die Generalkommission der Gewerkschaften Deutschlands gewählt. ${ }^{71}$ Schmidt hatte sich besonders in der Maifeierfrage engagiert. Er führte gegen dem Widerstand Legiens den Beschluß herbei, da $ß$ diese Frage auf dem Kölner Kongreß behandelt würde ${ }^{72}$ und vertrat auf dem Kongreß die Resolution der Generalkommission, die für Abendveranstaltungen am 1. Mai statt Arbeitsruhe plädierte. ${ }^{73}$

August Bebel war seit der Gründung der Sozialdemokratischen Arbeiterpartei 1869 einer der Führer der Sozialdemokratie und seit 1890 Vorsitzender der SPD. Seine Führungsposition in der deutschen Sozialdemokratie war zum Zeitpunkt dieses Briefwechsels absolut unumstritten, die ihm aus der Partei entgegengebrachte Hochachtung grenzte oft an Verehrung. In den strittigen Fragen Maifeier und Massenstreik hatte sich Bebel bis zum Jenaer Parteitag nicht exponiert, aus seinen Briefen geht hervor, daß er sich mit dem Problem des Massenstreiks intensiver erst im August-September 1905 beschäftigte. ${ }^{74}$

Die in Bebels Antwortschreiben an Schmidt ${ }^{75}$ vorgeschlagene Zusammenkunft von Parteivorstand und Generalkommission erbrachte zumindest in der Maifeierfrage konkrete Ergebnisse. Die vom Antragsrat zur Maifeier dem Parteitag vorgelegte Resolution Fischer ${ }^{76}$ wurde ausdrücklich mit voller Zustimmung der Generalkommission präsentiert. ${ }^{77}$ Schmidts

\footnotetext{
69 NachlaB Bebel B 153, Internationaal Instituut voor Sociale Geschiedenis, Amsterdam.

$70 \mathrm{Vgl}$. Protokoll Jena, S. 312, 318, 337.

71 Vgl. Schönhoven, Expansion und Konzentration, a.a.O., S. 306.

72 Protokoll Vorständekonferenz 1904, S 4-7.

73 Protokoll Köln, S. 229ff.

74 Bebel an Kautsky, 1. August 1905, in August Bebels Briefwechsel mit Karl Kautsky, hrsg. von K. Kautsky Jr, Assen 1971, S. 170; ders, an Adler, 16. September, a.a.O.

75 Nachlaß Bebel B 44.

76 Protokoll Jena, S. 141.

$n$ Ebd., S. 241, 263.
} 
weitgehende finanzielle Forderungen wurden dem Parteitag in dieser Form nicht vorgelegt, jedoch räumte der Referent Fischer ein:

Wenn wir wirklich die Agitation für die Maifeier in dem Sinne betreiben, um schließlich die gesamte Arbeitsruhe zu ermöglichen, dann wird uns das wahrscheinlich schwere Opfer kosten. Daß diese Opfer den Gewerkschaften allein nicht aufgebürdet werden können, darüber sind wir uns klar. [ . . ] Die Partei ist nicht so kleinlich, sie würde auch, wenn die Maifeier große Opfer erforderte, keinen Augenblick zögern, mit vollen Händen zu geben, genau so wie die Gewerkschaften. ${ }^{78}$

Die gegenüber der Amsterdamer Maifeierresolution wesentlich großzügiger gefaßte Einschränkung der Jenaer Resolution ${ }^{79}$ darf man als aus der Besprechung resultierendes Zugeständnis an die Generalkommission ansehen. Die Generalkommission gab ihr Einverständnis mit der Resolution allerdings nur unter dem Vorbehalt, daß die Maifeierfrage damit nicht endgültig, sondern nur vorläufig - nämlich bis zum nächsten internationalen Kongreß - geregelt sei ${ }^{80}$ Legien äußerte später die Ansicht, daß es dieser Vorbesprechung zu verdanken gewesen wäre, ,,daß in dem Referat über Generalstreik die Angriffe gegen die Gewerkschaften nicht in ähnlicher Weise gekommen sind wie vorher in der Parteipresse" ${ }^{81}$ Ähnlich sah dies der Sekretär des Parteivorstands Hermann Molkenbuhr, der nach dem Parteitag notierte: ,,So ist denn der Parieitag in Jena ganz anders verlaufen, als viele gedacht haben. Wäre doch die Geschlossenheit der Partei, die hier geheuchelt wurde, in Wirklichkeit vorhanden, d.h., wären die sogenannten Führer so einig wie die Genossen."

Der Brief Schmidts wurde mit Maschine geschrieben und umfaßt drei doppelseitig beschriebene Blätter sowie ein einseitig beschriebenes Blatt. Nach Abfassung des Briefes wurden handschriftliche Ergänzungen und Korrekturen vorgenommen, Bebels Antwortschreiben wurde handschriftlich auf drei Seiten abgefaßt. Die Wiedergabe der beiden Schreiben folgt in der Schreibweise den Originalen.

78 Ebd., S. 276.

79 Die Arbeiter wurden zur Arbeitsruhe aufgefordert ,,überall da, wo die Möglichkeit der Arbeitsruhe vorhanden ist", ebd., S. 141. Demgegenüber hieß es in Amsterdam: ,,überall dort, wo es ohne Schädigung der Arbeiterinteressen möglich ist", Correspondenzblatt, Jg. 14, Nr 36, S. 596.

80 Correspondenzblatt, Jg. 15, Nr 46, S. 763 (Redaktionelle Anmerkung).

81 Protokoll der Konferenz der Vertreter der Vorstände der Zentralverbände, abgehalten im Berliner Gewerkschaftshaus vom 19. bis 23. Februar 1906, S. 47.

82 Tagebuchnotiz H. Molkenbuhrs, datiert 23. September 1905, Nachlaß Hermann Molkenbuhr, Kassette 4, Tagebuch 1905-09, Archiv der sozialen Demokratie, Bonn. 


\section{SCHMIDT AN BEBEL}

9. August $1905^{\mathrm{a}}$

Brief an Bebel ${ }^{b}$

Werter Genosse!

Wie Ihnen seitens des Parteivorstandes schon mitgeteilt sein dürfte, hat die Generalkommission den Wunsch geäussert, dass vor dem Parteitag mit beiden Körperschaften nochmals eine Besprechung stattfinde, um gewisse Differenzpunkte auszugleichen. Mir persönlich wäre es sehr angenehm, wenn Sie der Sitzung beiwohnen könnten, da ja sonst die Aussprache weniger Wert hätte. Ich hoffe, dass auch Genosse Singer ${ }^{83}$ bis zu der Zeit zurück ist, um ebenfalls an der Besprechung teilnehmen zu können.

Diese Besprechung ist meinerseits angeregt, und ich möchte Ihnen deshalb kurz die Gesichtspunkte darlegen, die mich veranlassten, diese Aussprache herbeizuführen.

Es erscheint mir dringend notwendig, trotz der zum Teil unverständlichen Angriffe auf die Gewerkschaftsbewegung, die in der Parteipresse laut geworden sind ${ }^{84}$ Auseinandersetzungen auf dem Parteitag zu vermeiden. Einmal sind diese Auseinandersetzungen meiner Auffassung nach zwecklos, sodann würden sie nur zum Gaudium der Gegner und der bürgerlichen Partei geschehen.

Ich muß allerdings sagen, dass ich es bisher nicht für möglich gehalten habe, dass von der Partei eine so schiefe Auffassung über die Gewerkschaftsbewegung ${ }^{c}$ vertreten werden könnte, als es tatsächlich geschehen ist. Ich brauche nur zu erinnern an die ganz haltlosen, unmotivierten Angriffe gegen die Tarifvereinbarungen, die von den Gewerkschaften gepflogen werden, ${ }^{85}$ und an die übertriebenen Angriffe gegen den Neutralitätsstand-

\section{a 1905 dem Datum mit Bleistift angefügt \\ b mit Bleistift angemerkt \\ c über die Gewerkschaftsbewegung nachträglich mit Bleistift eingefügt}

83 Paul Singer (1844-1911) war auf dem Parteitag in Halle 1890 gemeinsam mit Bebel zum Parteivorsitzenden gewählt worden. Nominell waren beide Parteivorsitzende gleichberechtigt, tatsächlich war Bebels Einfluß auf die Partei wesentlich größer. Singer vertrat seit 1884 den 4. Berliner Wahlkreis im Reichstag.

84 Siehe oben, S. 74f.

85 Nachdem im November 1904 in der Neuen Zeit ein Artikel erschienen war, der die zunehmenden Abschlüsse von Tarifverträgen als Erneuerung des Zunftgedankens sah und dies positiv bewertete (B. Schildbach, ,,Der Zunftgedanke im Tarifvertrag", in: Neue Zeit, Jg. 23, Bd 1, S. 204-10), erschienen mehrere Artikel, die auf die den Tarifverträgen inhärenten Gefahren hinwiesen (F. Schnetter, ,Der Zunftgedanke im Tarifvertrag”, ebd., S. 659-64; P. Umbreit, ,,Gewerbliche Friedensschwärmerei”, ebd., Bd 2, S. 45-54). Während diese Artikel nur auf die Gefahr gewerkschaftlicher Stagnation 
punkt. ${ }^{86}$ Ich glaube, Sie werden mit mir der Auffassung sein, dass die Gewerkschaften, wie die Dinge heute liegen, gezwungen sind, eine gewisse Neutralität aufrecht zu erhalten. - Es mag sein, dass im Bergarbeiterverband von Hue und Leimpeters ${ }^{87}$ der Neutralitätsstandpunkt oft in übertriebener Weise vertreten ${ }^{\mathrm{d}}$ wurde. Indes sind gerade beim Bergarbeiterverband die eigentümlichen Verhältnisse zu berücksichtigen, die Schwierigkeit der Agitation und des Vorwärtskommens gegen den Einfluss des Centrums, sodass, wenn hier übers Ziel geschossen wird, die Taktik dennoch verständlich und meiner festen Ueberzeugung nach zum Schaden der Partei nicht ausgeartet ist. Natürlich ist es unausbleiblich, wie sich das bei dem großen Bergarbeiterstreik ${ }^{88}$ gezeigt hat, dass hier und da von einigen ungeübten Leuten über das Mass der notwendigen Zurückhaltung der politischen Bewegung gegenüber hinausgegangen wird. Indes, diese an und für sich unbedeutenden Vorgänge kann man nicht, wie das die Dortmunder Arbeiterzeitung getan hat, schliesslich dem Gesamtverbande zur Last legen. ${ }^{89}$

\section{d zunächst übertreten dann mit Bleistift geändert}

hinwiesen, ohne Tarifverträge abzulehnen, zählte Kautsky die Tarifverträge zu den Faktoren, die keinen Fortschritt der Gewerkschaften bewirkten, , sondern eine Tendenz zum Beharren beim Errungenen" (Die Lehren des Bergarbeiterstreiks", ebd., Bd 1, S. 776).

86 Etwa seit 1900 war die Frage umstritten, inwieweit sich die Gewerkschaften parteipolitisch neutral verhalten sollten, um unpolitische Arbeiter in ihre Organisationen zu ziehen.

87 Otto Hue (1868-1922), Zechenschlosser, war seit 1895 Redakteur der BergarbeiterZeitung, seit 1903 Mitglied des Reichstags. Obwohl er Redakteur blieb, wurde er bald der eigentliche Führer der Bergarbeitergewerkschaft (vgl. Neue Deutsche Biographie, Bd 9, S. 716f.) und trat wegen der im Ruhrgebiet großen Anhängerschaft des christlichen Bergarbeitervereins für weitgehende parteipolitische Neutralität des ,Alten Verbands" ein. Leimpeters (siehe oben, S. 75) war ebenfalls Redakteur der Bergarbeiter-Zeitung. Ihm wurde in der Generalversammlung der Bergarbeiter-Gewerkschaft im Juni 1905 vorgeworfen, er ,, apportiere" meist die Ansichten von Hue (vgl. Vorwärts, Jg. 22, Nr 136, S. 6).

88 Gemeint ist der Bergarbeiterstreik im Ruhrgebiet, der am 7. Januar 1905 begann und am 9. Februar abgebrochen wurde. Dabei waren über 200000 Bergarbeiter beteiligt. Vgl. D. Fricke, Der Ruhrbergarbeiterstreik von 1905, Berlin 1955; O. Hue, ,,Über den Generalstreik im Ruhrgebiet", in: Sozialistische Monatshefte, Jg. 9, Bd 1, S. 201-10.

89 Die Zusammenarbeit des Bergarbeiterverbands mit der christlichen, der HirschDuncker'schen und der polnischen Gewerkschaft im Ruhrgebiet während des Bergarbeiterstreiks war auf Kritik gestoßen. Besonders das lokale Parteiblatt Dortmunder Arbeiterzeitung bzw. deren Redakteur Konrad Haenisch warf dem Verband vor, durch „,Hyperneutralität” der Sache der Sozialdemokratie geschadet zu haben. Der Verband habe den Streik nicht genügend zu sozialdemokratischer Agitation genützt. Statt mit den anderen Gewerkschaften zu kooperieren, hätte der Alte Verband sich an die Spitze des 
Ich habe durch meine persönlichen Beziehungen den Eindruck, dass in der Verwaltung des Bergarbeiterverbandes durchaus überzeugte und zum Teil recht eifrige Parteigenossen vorhanden sind.

Ich schreibe das alles, weil ich von Ihrer letzten Rede in der Berliner Zusammenkunft ${ }^{90}$ den Eindruck gewann, dass auch bei Ihnen sich Ansichten und Anschauungen über Partei und Gewerkschaft festgesetzt haben, die meiner Ueberzeugung nach auf einseitiger Information beruhen. In der Sitzung selbst war die Zeit schon zu weit vorgeschritten, um auf all die Dinge zurückkommen zu können. So gaben Sie in der Sitzung an, dass in Hamburg die Parteigenossen die wundersamsten Dinge über Tätigkeit und Anschauungen der Gewerkschaftsführer Ihnen mitgeteilt hätten. ${ }^{91}$

Ich möchte bitten, dabei zu berücksichtigen, dass seit Jahren gerade in Hamburg zwischen einigen leitenden Personen der Gewerkschaftsbewegung, dem ,Echo"92 und der Partei Differenzen vorhanden sind. Daraus erklären sich gewisse persönliche Gereiztheiten und persönliche Abneigung, die sich dann schliesslich verallgemeinert und zur Abneigung auf der einen Seite gegen die Partei, auf der anderen Seite gegen die Gewerkschaft

Streiks stellen sollen, um so die Arbeiter von den christlichen Gewerkschaften wegzuziehen. Ein weiterer Vorwurf der Arbeiterzeitung richtete sich gegen den Abbruch des Streiks, der ohne Rücksicht auf die kämpferische Stimmung der Massen geschehen sei. Vgl. Correspondenzblatt, Jg. 15, S. 98-100, 113-15; Vorwärts, Jg. 22, Nr 136, S. 5; O. Hue, ,Über den Generalstreik im Ruhrgebiet”, S. $207 \mathrm{f}$.

90 Am 17. Juni 1905 traten auf Einladung der Bertiner Preßkommission - die in Gemeinschaft mit dem Parteivorstand die Angelegenheiten des Vorwärts verwaltete und kontrollierte - die Vertrauensleute Berlins, die Preßkommission, die Lokalkommission, die Agitationskommission für die Provinz Brandenburg, der Parteivorstand und die Vertreter Berlins im Reichstag zu einer gemeinsamen Sitzung zusammen, bei der über die politische Haltung des Vorwärts debattiert wurde. Dem Vorwärts wurde ein einstimmiges Mißtrauensvotum ausgesprochen. Bebel als Mitglied des Parteivorstands und Schmidt als Vertreter des fünften Wahlkreises gehörten zum Kreis der Eingeladenen (vgl. Protokoll Jena, S. 187; G. Ledebour, ,,Eine Literatenrevolte”, in: Neue Zeit, Jg. 24 (1905-06), Bd 1, S. 189-94). Ein anderer Bezug erscheint unwahrscheinlich, da Bebel am 19. Juni nach Zürich abreiste. Vgl. Bebel an Adler, 30. Juni, in Victor Adlers Briefwechsel, a.a.O., S. 458. Dazu auch ders. an Kautsky, 1. August, a.a.O., S. 170f. Hier liegt ein - bei Bebels Handschrift verständlicher - Irrtum vor. Die Ortsangabe Breslau in der letzten Zeile auf S. 170 entspringt wohl einem Lesefehler, richtig heißt es Berlin.

91 Anläßlich der in Hamburg geplanten Wahlrechtsveränderung, die der Sozialdemokratie das Erreichen einer Mehrheit in der Bürgerschaft unmöglich machen sollte (vgl. dazu O. Stolten, , Der geplante Wahlrechtsumsturz in Hamburg”, in: Neue Zeit, Jg. 23, Bd 2, S. 322-27), hatte Ende Mai 1905 in Hamburg eine Konferenz der lokalen Parteivorstände und der Hamburger Gewerkschaftskommission stattgefunden, bei der dem teilnehmenden Bebel von seiten älterer Gewerkschaftler gesagt wurde, ein Teil jüngerer Gewerkschaftsführer in Hamburg stünde nicht auf dem Boden der Sozialdemokratie, höhne über den Sozialismus und bestreite, daß man einen Klassenkampf führe. Vgl. Protokoll Jena, S. 308.

92 Gemeint ist das Parteiorgan Hamburger Echo. 
führt. ${ }^{93}$ Es wird sicherlich in der Beziehung auf beiden Seiten gesündigt; aber weit gefehlt wäre es, allgemein zu behaupten, dass die Gewerkschaften der Parteibewegung irgendwie hindernd im Wege ständen. Ich habe die feste Ueberzeugung, dass die ganzen Fortschritte der Parteibewegung, beispielsweise in Oberschlesien, der Gewerkschaftsagitation verdankt werdene.

Schon die zahlreich angestellten Beamten geben der Bewegung in solchen Gegenden ${ }^{\mathrm{f}}$ einen ganz anderen Rückhalt, als wenn durch gelegentliche Agitationen die Massen aufgerüttelt werden.

Ob man den Neutralitätsstandpunkt der Gewerkschaften betont oder nicht, - in einem solchen Industriebezirk wie Oberschlesien führt die gewerkschaftliche Organisation die Arbeiter auch zur politischen Betätigung.

Aehnlich wie in Hamburg liegen zum Teil auch die Dinge, wenn auch nicht so krass, in Berlin.

Aus mir eigentlich unbegreiflichen Gründen - und ich darf sagen, ich habe sowohl mit der Partei, als mit der Gewerkschaft sehr viele Beziehungen - herrscht unter einer Anzahl Parteigenossen eine ganz unerklärliche Animosität gegen die Gewerkschaft. Ich möchte nur auf eines hinweisen, was so ganz bezeichnend ist für die Haltung der leitenden Personen in Berlin.

Es ist bekannt, dass das Gewerkschaftshaus nicht gerade glänzend steht. Trotzdem wird systematisch vom dritten Wahlkreis die Abhaltung von Versammlungen in anderen Lokalen betrieben.

Ich möchte behaupten, dass das Gewerkschaftshaus nahezu boykottiert wirds. - Bei der vorigen Maifeier bedurfte es erst sehr energischer Vorstellungen, dass im Gewerkschaftshaus eine Versammlung abgehalten wurde. Auch bei den letzten Versammlungen über Fleischteuerung ist das Gewerkschaftshaus ausser acht gelassen worden. -

Man muss doch schließlich zugeben, dass in einem Hause, das von den Berliner Arbeitern gebaut wurde, zu allererst hier die Stätte ist, wo die

e zunächst wird dann mit Bleistift geändert

f ursprünglich in solchem Falle dann mit Bleistift korrigiert; Lesart Gegenden nicht eindeutig, möglicherweise Flüchtigkeitsfehler

$\mathrm{g}$ wird nachträglich mit Bleistift eingefügt

93 Ähnlich wie Schmidt bestritt auf dem Parteitag der Hamburger Gewerkschaftler v. Elm (vgl. Anm. 63), daß eine parteifeindliche Stimmung in Hamburger Gewerkschaftskreisen herrsche. Auch er führte dieses Gerücht auf persönliche Differenzen zurück: ,Vielleicht gibt es aber einige Leute in Hamburg, die ihre werte Persönlichkeit mit der Partei identifizieren, die, wenn sie angegriffen werden, meinen, die Partei sei gehöhnt." Protokoll Jena, S. 331. 
Arbeiter zu demonstrieren und Versammlungen abzuhalten haben. Das sind auch so einige Vorgänge, die Gegensätze hervorrufen.

Auf dem letzten Gewerkschaftskongress ist mir von dem Genossen Hueber ${ }^{94}$ in Bezug auf die Vorgänge in Köln gesagt worden, ein ähnlicher Vorgang wäre in Oesterreich nicht möglich, weil dort die leitenden Personen d. Gewerksch. und die Partei viel zu enge Beziehungen zu einander unterhielten.

Ich habe den Eindruck, dass diese Bemerkung des Genossen Hueber nicht ganz unberechtigt ist. Es ist mir die Berechtigung des Ausspruches noch $^{\mathrm{h}}$ mehr zum Bewusstsein gekommen ${ }^{\mathrm{i}}$ nach Ihrer letzten Rede in der Berliner Zusammenkunft.

Ich möchte mir deshalb einen Vorschlag erlauben, der vielleicht Aussicht auf Verwirklichung haben könnte.

Wir werden nicht dazu kommen können, einen Vertreter der Generalkommission in den Parteivorstand zu wählen. Es lässt sich sicherlich an dem gegenwärtigen Zustand ${ }^{j}$ auch nichts ändern. Ich glaube aber, es wäre möglich, in die Controllkommission durch stillschweigende Vereinbarung ein Mitglied der Generalkommission hineinzuwählen. Manche Vorurteile könnten auf diesem Wege sofort beseitigt und unrichtige Informationen richtig gestellt werden. ${ }^{95}$

Ueber eine geeignete Person liesse sich vielleicht bei der Besprechung, event. auf dem Parteitag, eine Verständigung herbeiführen.

Bei der Besprechung liegt mir daran, die Differenzen wegen der Maifeier zu beseitigen. Ich denke mir die Regelung der Angelegenheit so, dass der Parteivorstand sich bereit erklärt, auf dem Parteitag einen Antrag zu

${ }^{\mathrm{h}}$ nun gestrichen, stattdessen noch mit Bleistift eingefügt

nach gekommen wurde als gestrichen

i nach Zustand wurde der gestrichen

94 Anton Hueber (1861-1935) war als Vertreter der österreichischen Gewerkschaften Gast des Kölner Kongresses. Der gelernte Drechsler Hueber war im Januar 1895 zum Sekretär der österreichischen Reichsgewerkschaftskommission gewählt worden, die er in der Folgezeit anführte. Auf dem Parteitag der österreichischen Sozialdemokratie 1894 war er als entschiedener Vertreter des Massenstreiks aufgetreten, womit er sich in Gegensatz zu Victor Adler setzte, welcher sich letztlich durchsetzen konnte. Später rückten dann Partei- und Gewerkschaftsführung politisch so nahe zusammen, daß Adler und Hueber oft scherzhaft als ,,Siamesische Zwillinge" bezeichnet wurden. Vgl. Große Österreicher. Neue Österreichische Biographie ab 1815, Bd 14, S. 122-28.

${ }_{95}$ Wie sich Schmidt diesen Vorgang vorstellte, erscheint unklar. Da die Kontrollkommission ebenso wie der Parteivorstand auf den Parteitagen gewählt wurde, wäre das entsprechende Mitglied der Generalkommission nicht daran vorbeigekommen, sich zur Wahl stellen zu müssen. Eine ,,stillschweigende Vereinbarung" im Sinne einer automatischen Kooptierung wäre ein schwerer Verstoß gegen die Regeln der innerparteilichen Demokratie gewesen, der nicht ohne weiteres toleriert worden wäre. 
vertreten, der besagen würde, dass die infolge der Maifeier gemassregelten Arbeiter, soweit sie von Gewerkschaften keine Unterstützungen erhalten, von der Partei in entsprechender Weise unterstützt werden. Es würden dazu im wesentlichen die Unorganisierten gehören, sowie auch diejenigen, die bei hohen Ansprüchen an die Organisation von der Gewerkschaft nicht mehr unterstützt werden können. ${ }^{96}$ Sodann würde ich der Generalkommission empfehlen, sie erklärt ${ }^{k}$ nach der hier ${ }^{l}$ gekennzeichneten Vereinbarung nunmehr, ihr Vorhaben bezüglich einer Aenderung in der Maifeier aufzugeben $^{\mathrm{m}}$. Damit würde auf dem Parteitag meiner Ansicht nach einer sehr unliebsamen Debatte von vornherein die Schärfe genommen" und wir hätten nicht Vorgänge zu verzeichnen, wie sie sich vor zehn Jahren in Köln auf dem Parteitag abgespielt haben. ${ }^{97}$

Wie die Dinge heute liegen, besteht gar kein Zweifel, dass, wenn von unserer Seite - damit meine ich den Parteivorstand und die Generalkommission - nichts geschieht, um den Zwist im Keim zu ersticken, die Verhandlungen in Jena sicherlich für beide Teile keinen erfreulichen Ausgang nehmen werden.

Wir haben sicherlich in der Partei und Gewerkschaftsbewegung jetzt wichtigere Dinge zu tun, als uns gegenseitig zu raufen. -

In der Frage des Generalstreiks wird meiner Ansicht nach allerdings eine Verständigung nicht herbeizuführen sein.

Die Frage ist von so grosser Bedeutung für die künftige Taktik der Partei, dass hier ein Ueberbrücken der Gegensätze, die nicht nur bestehen zwi-

k mit Bleistift korrigiert, erklärt statt erkläre

' hier mit Bleistift geschrieben

m Bleistiftkorrektur: nach Maifeier wurde für gestrichen, aufgegeben wurde geändert in aufzugeben

n nach genommen wurde werden gestrichen

96 Den Gedanken einer finanziellen Beteiligung des Parteivorstandes an den Kosten der Maifeier hatte bereits im Vorjahr - anscheinend ohne Absprache mit dem gesamten Vorstand - der Sekretär des Parteivorstands Pfannkuch geäußert (Protokoll Vorstandskonferenz 1904, S. 4f.), was bei der Gewerkschaftsführung auf Beachtung stieB. Wenn Schmidts Modell der Kostenverteilung auf Partei und Gewerkschaft in Jena auch keine Umsetzung erfuhr (vgl. oben, S. 77f.), so blieb sein Grundgedanke für die Maifeierdiskussion der nächsten Jahre doch bestimmend. $\mathrm{DaB}$ die Unterstützung der wegen der Maifeier gemaßregelten Arbeiter gemeinsam von Partei und Gewerkschaft getragen werden sollte, wurde dann zum ersten Mal von der deutschen Delegation beim Kongreß der Internationale in Stuttgart 1907 beschlossen (vgl. Correspondenzblatt, Jg. 17 (1907), S. 554).

97 Der hier angesprochene Kölner Parteitag lag tatsächlich schon 12 Jahre zurück. Der Parteitag 1893 hatte den damals in ihrer Position schwer erschütterten Gewerkschaften die volle Unterstützung der Partei versagt. Dieser Parteitag brachte eine Vertrauenskrise zwischen Partei und Gewerkschaft hervor. 
schen Partei und Gewerkschaft, sondern vor allem auch in der Partei, unmöglich ist. Ich denke aber, wenn wir den voraufgegangen $Z$ wist beseitigen, wird diese Debatte zu einer ruhigeren Auseinandersetzung führen, als es sonst der Fall sein könnte. -

Ich darf wohl annehmen, dass Ihnen wie mir daran gelegen sein wird, den Versuch zu unternehmen, die von mir bezeichneten Differenzen vor dem Parteitag auszugleichen, damit wir nicht auf jedem Parteitag der Aussenwelt das Beispiel innerer Zwistigkeiten bieten.$^{98} \mathrm{Zu}$ dem Zweck würde ich, wie eingangs erwähnt, es sehr gern sehen, wenn Sie es ermöglichen könnten, bei der Conferenz zugegen zu sein.

Besten Gruss!

Robert Schmidt

\section{BEBEL AN SCHMIDT}

Zürich, den 15. Aug. 1905

Usteristr. $14^{99}$

Werter Genosse Schmidt!

Ihr Brief vom 9. kam in meine Hände. Schrieb ich nicht eher, so weil wir vor der Abreise von Zug ${ }^{100}$ nach hier standen.

Ich bin selbstverständlich gern bereit, in eine Aussprache zwischen dem Parteivorstand und der Generalkommission zu willigen. Ich schlage vor, daß wir dieselbe in Jena, Samstag Abend den 16. Sept. führen. ${ }^{101}$ Am

98 Der heftige Revisionismusstreit auf dem Dresdener Parteitag 1903 war 1905 noch in bester Erinnerung. Das satirische Blatt Kladderadatsch, Jg. 58 (1905), Nr 39, zeigte sich z.B. sehr enttäuscht über den ruhigen Verlauf des Jenaer Parteitags: ,, Wie groß einst zeigte die Partei sich / Die Bebel führt, wie sprach sie frei sich/In Dresden aus mit offnem Sinn! / Ja, groß war sie am Strand der Elbe / Doch heut ist sie nicht mehr dieselbe / Obgleich zwei Jahre erst dahin. [. . .] Nicht droht man seinem Gegner Keile / Mehr an, die graue Langeweile / Führt's Regiment im Saale jetzt / Und wagt einmal ein alter Kämpfer / Ein freies Wort, so wird ein Dämpfer / Von Singer schleunigst draufgesetzt." 99 Bebel besaß ein Haus in Zürich, das ihm vererbt worden war. Vgl. F. Osterroth, Biographisches Lexikon des Sozialismus, Bd 1, Hannover 1960, S. 18.

100 Zug, die Hauptstadt des gleichnamigen Schweizer Kantons, liegt ca $30 \mathrm{~km}$ südlich von Zürich. Bebel pflegte sich, seitdem er das Züricher Haus besaß, im Sommer für einige Wochen zur Erholung in der Schweiz aufzuhalten (vgl. H. Wendel, August Bebel, Eine Lebensskizze, Offenbach 1948, S. 103). Im Sommer 1905 reiste Bebel am 19. Juni nach Zürich (an Adler, 30. Juni, a.a.O.); von dort suchte er eine internationale Versammlung in Konstanz am 9. Juli auf (ebd.; Protokoll Jena, S. 35ff.). Nach einem erneuten Aufenthalt in Zürich reiste Bebel dann - etwa in der Mitte des Juni - nach Zug, wo er sich zur Erholung bis zum 14. August aufhielt (an Kautsky, 1. August, S. 169f.). 101 Der Jenaer Parteitag der SPD wurde am 17. September um 19 Uhr eröffnet, Protokoll Jena, S. 145. 
Sonntag den 17. haben wir keine Zeit, da wir den ganzen Tag Sitzung haben (Vorstand u. Controllkommission). Gleichzeitig könnten an dieser Besprechung auch die anwesenden Mitglieder der Contr.Kommission u. der Antr.rat über die Maifeier ${ }^{102}$ teil nehmen.

Vorher nach Berlin zu kommen, ist mir nicht möglich. Mein Hausstand ist vorläufig aufgelöst, außerdem habe ich vor dem Parteitag noch einige Orte in Süddeutschland zu besuchen, so daß ich erst am Abend des 16. in Jena eintreffen kann. Verständigen Sie sich mit dem Vorstand über die Zusammenkunft, die etwa um $8 \mathrm{Uhr}$ stattfinden könnte. Ich treffe um $6 \mathrm{Uhr}$ ein.

Ich will mich in Anbetracht dieser Aussprache hier über den Inhalt Ihres Briefes nicht auslassen. Ich glaube, er geht vielfach von falschen Voraussetzungen $u$. vermutlich auch von Mißverständnißen in Bezug auf meine Person und meine Auffassungen aus.

Ich bin mit Ihnen der Meinung, daß Partei \& Gewerkschaft nicht die geringste Ursache haben, sich zu befehden, daß wir, die Partei, alle Ursache haben, den Gewerkschaften die rasche Entwicklung zu wünschen u. nach Kräften dazu beizutragen; daß aber auch das Umgekehrte der Fall sein soll $u$. daß in diesem Punkte hie $u$. da gesündigt wurde.

Daß eine gewisse Entfremdung zwischen der Gen.Kommission und dem Parteivorstand besteht, ist zum geringsten Teil unsere Schuld. Sie wollen doch festhalten, daß wir es waren, die vor dem Intern. Kongre $\beta^{103} \mathbf{u}$. in der Fraktion $^{\circ}$ Verständigung suchten. Daß die Tagesordnung des Köln. Gew. Congreßes festgestellt wurde ohne ein Wort der Benachrichtigung, obwohl die Partei aufs lebhafteste daran interessiert war.

Auch ich bin der Ansicht, daß gleiches in Oesterreich unmöglich wäre u. unmöglich ist.

Die Animosität gegen das Gew.Haus ist mir neu. ich brauche nicht zu sagen, daß ich dieses mißbillige \& bedaure u. für meinen Teil beitragen werde zu verhüten, daß Vorkommniße, wie Sie die selben schildern, sich eräussern.

- u. in der Fraktion nachträglich eingefügt

102 Aus der Formulierung ,,Antragsrat" geht hervor, daß die Jenaer Maifeierresolution nicht von dem Referenten Richard Fischer allein, sondern von einer Kommission entworfen wurde. Dies wird aus dem Parteitagsprotokoll nicht ersichtlich und weist darauf hin, welche Bedeutung diesem Tagesordnungspunkt beigemessen wurde.

103 Über die Verhandlungen von Parteivorstand und Generalkommission vor dem Amsterdamer Kongreß 1904 siehe Einleitung. Möglicherweise war die in der Maifeierresolution enthaltene Einschränkung (siehe ebd.) bereits ein aus den Verhandlungen resultierendes Entgegenkommen der Parteiführung an die Wünsche der Generalkommission. 
Ueber die Art, wie intensive Beziehungen zwischen den Leitern der beiden Gruppen der Arb. Bewegung herbeigeführt werden können, mündlich mehr. Der von Ihnen gemachte Vorschlag erreicht nicht den Zweck. ${ }^{104}$ Ist die Absicht auf der Gegenseite zu freundschaftlicher Verständigung vorhanden, so wird sie nicht über Mangel an Entgegenkommen auf unserer Seite zu klagen haben.

Mit Parteigruß

A. Bebel

104 Für die hier von Bebel abgelehnte Idee einer personellen Verklammerung von Partei- und Gewerkschaftsführung trat zur gleichen Zeit Karl Kautsky in einem Brief an Victor Adler ein. Er vermutete, daß der Parteivorstand aber für diese Idee nicht zu gewinnen wäre, weil deren Realisation bedeuten würde, daß man sich zusätzlich um Gewerkschaftsangelegenheiten kümmern müßte: „,Freilich, beim Parteivorstand werden Vorschläge auf Erweiterung seines Arbeitsgebiets nicht auf viel Gegenliebe stoßen. Er ist ein Kollegium alter Herren, die von den Bürogeschäften und dem Parlamentarismus so absorbiert werden, daß sie jede Erweiterung ihres Arbeitsgebiets verfluchen. Sich um Gewerkschaften und Konsumvereine zu kümmern, fällt ihnen nicht ein: das fehlte gerade noch, meinte einmal Singer zu mir, als ich ihm davon sprach." Adler, 2. August 1905, in Victor Adlers Briefwechsel, S. 467. 